\title{
Miniaturization in Glaucoma Monitoring and Treatment: A Review of New Technologies That Require a Minimal Surgical Approach
}

\author{
H. Burkhard Dick - Tim Schultz - Ronald D. Gerste
}

Received: April 13, 2018 / Published online: February 6, 2019

(C) The Author(s) 2019

\section{ABSTRACT}

In the management of glaucoma, recent and upcoming innovations have the potential to contribute to both the efficacy of intraocular pressure (IOP) monitoring and the number of available treatment options. These new devices and procedures have two things in common: they are part of the trend in medicine towards miniaturization, and they require a limited surgical procedure to become effective. This review focuses on the Eyemate (Argos) intraocular sensor, which offers a new way to reliably measure $24 \mathrm{~h}$ IOP, and on intraocular sustained release systems for pharmacological glaucoma therapy. It also briefly reflects on the miniature implants currently used in minimally invasive glaucoma surgery (MIGS).

Keywords: Adherence; CyPass microstent; Eyemate (Argos) sensor; Glaucoma; Hydrus microstent; Intraocular drug delivery systems; Intraocular pressure (IOP); iStent inject;

Enhanced Digital Features To view enhanced digital features for this article go to https://doi.org/10.6084/ m9.figshare.7388321.

H. B. Dick - T. Schultz - R. D. Gerste ( $₫)$

University Eye Clinic, Ruhr University, Bochum, Germany

e-mail: rdgerste@aol.com
Minimally invasive glaucoma surgery (MIGS); XEN gel

\section{INTRODUCTION}

Glaucoma is considered the second most frequent cause of global blindness. The most common form of the disease, primary openangle glaucoma (POAG), afflicts nearly $75 \%$ of the projected 80 million glaucoma patients worldwide [37].

In the industrialized world, treating glaucoma and monitoring glaucoma patients is a mainstay of daily ophthalmological practice. POAG particularly affects the elderly, as the risk of suffering from glaucoma increases with age. In Americans aged 40 years or older, glaucoma prevalence is about $2.1 \%$ according to the National Health and Nutrition Examination Survey (NHANES). That study also confirmed a fact that is well known to every practitioner in eye care: that many people suffering from glaucoma are completely unaware of having the disease; in the NHANES, half of the glaucoma cases were previously undiagnosed [10].

In an elderly German population of 822 individuals aged 68-96 years, glaucoma was prevalent in $9 \%$ of the study participants-a proportion surpassed by only two other eye diseases, cataract (36\%) and dry eyes (15\%) [38]. Since most societies are undergoing demographic aging, the number of people suffering 
from glaucoma who are in need of glaucoma therapy and robust monitoring of their symptoms (particularly their intraocular pressure, IOP) is set to increase in the foreseeable future. In the United States, for instance, it is projected that the number of glaucoma patients will increase by $28 \%$ per decade [43].

\section{THE CHALLENGE OF MONITORING AND TREATING IOP IN THE REAL WORLD}

In recent years, considerable progress has been made in the detection, diagnosis, and treatment of glaucoma. Innovative imaging techniques, for instance, can highlight the early signs of glaucomatous damage and allow meticulous monitoring of the morphological changes that occur during the course of the disease and its progression. Our understanding of the pathomechanism of glaucoma has deepened, and the impact of factors such as oxidative stress and irregularities in retinal blood flow has been thoroughly investigated $[7,25,26]$. IOP is widely considered the only modifiable risk factor for glaucoma. Therefore, the disease is generally managed by lowering the pressure to slow the progression of a disease that cannot be cured but can-with the optimal approach-be controlled. Both monitoring the IOP and lowering it for therapeutic purposes entail a number of challenges in daily eye care practice, i.e., in the real world [20]. To get a clear picture of a patient's IOP, it is necessary to monitor diurnal and nocturnal variations ("dips and peaks"), which would require measurements far beyond those currently performed by most eye care providers on their patients, as such measurements are generally only carried out during visits to the doctor every 3 months. A one-off IOP measurement of this kind-probably taken at the same time of day at every visit, such as in the morning or after the office's lunch break-is nothing but a snapshot that provides hardly any information on what the pressure could be like 10 or $12 \mathrm{~h}$ later, during sleep, or when waking up in the morning.

In all three fields that comprise glaucoma management (diagnosis and long-term control based on reliable IOP measurements, the application of antiglaucomatous drugs, and surgical intervention), there have been some recent developments that seem to point to a future in which glaucoma monitoring and treatment will differ from the approaches used in the past and from what is considered routine or the "gold standard" today. These new devices and procedures have two things in common: they are part of the trend in medicine towards miniaturization-towards employing ever-smaller contraptions. And they are all (at least initially) handled by an ophthalmic surgeon-even the concept of "conservative" therapy described henceforth.

This is a compact overview of some developing and established technologies for glaucoma monitoring and treatment that fit the term "miniaturization." It does not aspire to review every aspect of this new and fascinating field. This article is based on previously conducted studies and does not contain any studies with human participants or animals performed by any of the authors. Table 1 presents a summary of miniaturized devices that have been developed for glaucoma management.

\section{DIAGNOSING AND MONITORING IOP}

Glaucoma is a 24-h disease, but eye care practices usually do not monitor IOP around the clock. This means that most long-term and short-term fluctuations in the IOP are not observed by traditional measurement regimes. Indeed, as recently as 4 years ago, an overview of the role of pressure fluctuations written by a group of authors from New York and Massachusetts concluded with the following slightly pessimistic statement: "Until a reliable method is developed that allows for constant IOP monitoring, many variables will continue to hinder us from drawing adequate conclusions regarding the significance of IOP variation" [18].

Aside from some other concepts that permit continuous IOP measurement, such as a contact lens that incorporates a sensor [12, 31, 41], the recently introduced and CE-certified (in Europe) Eyemate IOP sensor represents a reliable 
Table 1 A summary of the miniaturized devices used in glaucoma management (samples)

\section{Diagnostics}

$\begin{array}{cc}\text { Eyemate (Argos) } & \text { Wireless IOP sensor, } \\ \text { (Implandata, } & \text { implanted in sulcus } \\ \text { Hannover, Germany) } & \\ \text { Intraocular sustained antiglaucomatous drug release }\end{array}$

Bimatoprost SR Biodegradable implant

(Allergan)

ENV515 (Envisia Biodegradable implant

Therapeutics,

Durham, NC, USA)

iDose (Glaukos Corp., Titanium implant, to be

Laguna Hills, CA, replaced when depleted USA)

Drug delivery device Biodegradable material

by Amorphex

Therapeutics

\section{Minimally invasive glaucoma surgery (MIGS), implants}

Trabecular outflow

iStent (Glaukos Corp., FDA approved

Laguna Hills, CA,

USA)

iStent inject (Glaukos Corp., Laguna Hills, CA, USA)

Hydrus (Ivantis Inc., Investigational in US Irvine, CA, USA)

Uveoscleral outflow

$$
\text { CyPass (Alcon, Fort }
$$

FDA approved

CE marked

Worth, TX, USA) nvestigational in US, FDA

CE marked

$$
\begin{aligned}
& \text { Contains } \\
& \text { travoprost }
\end{aligned}
$$

Placed on the sclera underneath eyelid

CE marked approval expected for second half of 2018

\section{CE marked}
Placed in anterior chamber

Placed in anterior chamber
Placed in anterior chamber

Currently in phase 2a trials, containing a prostaglandin
Currently in phase III trials

Currently in phase 2 a trials

Phase 3 trial announced for 2018

$$
\begin{aligned}
& \text { Worldwide recall } \\
& \text { from market in } \\
& 2018 \text { due to } \\
& \text { safety concerns }
\end{aligned}
$$


Table 1 continued

Subconjunctival outflow

\begin{tabular}{lll} 
XEN Gel (Allergan, & Ab interno & FDA approved \\
Dublin, Ireland) & & \\
$\begin{array}{c}\text { InnFocus (Santen, } \\
\text { Osaka, Japan) }\end{array}$ & Ab externo & CE marked \\
\hline
\end{tabular}

Information in the table is accurate as of May 2018. The table is not intended to povide a full summary of all miniaturized devices used in glaucoma management. Data on approval or investigational status depends in most cases on information provided by the manufacturers

method of pressure monitoring that does not necessitate regular examinations in the office and requires only minimal patient cooperation. The Eyemate (also named the Argos) is a wireless IOP transducer that integrates pressure sensors, a temperature sensor, an identification encoder, an analog-to-digital encoder, and a telemetry unit into a single microelectromechanical system completely covered with biocompatible silicone (Implandata Ophthalmic Products GmbH, Hannover, Germany). This sensor is implanted into the sulcus (just like an intraocular lens, IOL), sometimes together with an IOL, during cataract surgery (Fig. 1).

Whether implanted during cataract surgery or in a standalone procedure, the Eyemate is designed to stay in the patient's eye indefinitely. The sensor does not require a battery. It derives the power it needs via electromagnetic inductive coupling to an external magnetic field

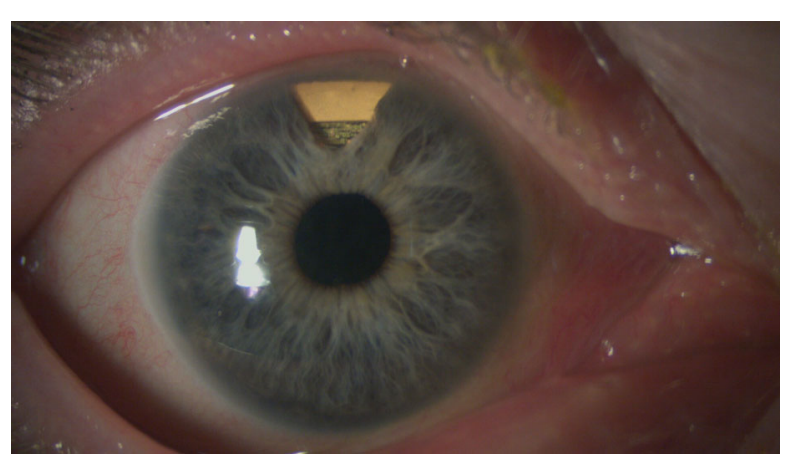

Fig. 1 The Eyemate sensor implanted, visible through the iridectomy at 12 o' clock generator housed in an external reader unit. The reader unit is battery powered and resembles a television remote control in its current design. The same reader unit picks up the digital data relayed by the transponder unit and subsequently displays the IOP values on its lightemitting diode display. The reader and the transponder unit need to be brought into close proximity with each other before a button is pressed on the reader to activate the electromagnetic coupling sequence-this is all the cooperation required from the patient. The sensor can conduct up to 10 measurements per second. The ophthalmologist receives the patient's IOP measurements by telemetry and can easily create the patient's tension profile, detect dips and peaks during diurnal and nocturnal IOP fluctuations, and recognize situations that require an adjustment to the therapy [23].

In an animal model, reliable IOP readings were possible without any human interaction. The device recorded the diurnal and nocturnal IOP-lowering effects of two antiglaucomatous drugs, latanoprost and dorzolamide [33]. The first clinical experiences with the sensor are encouraging. Though reports have thus far been anecdotal, implantation of the sensor went smoothly in our clinic, and the measurements, readings, and transmission of data were found to be reliable and uncomplicated [6]. The longterm safety of the intraocular sensor was confirmed in a small group of patients $(n=5)$ with an average follow-up of 37.5 months [17]. The intraocular sensor gives the eyecare provider a 
tool for noninvasively assessing the IOP at different times of the day (and night) and during different activities in the patient's daily life. Without any doubt, the analysis of these measurements and their impact on clinical practice requires more studies with larger groups of patients. It will be interesting to see how the IOP values documented by the sensor relate to Goldmann applanation tonometry, which is generally considered to be the gold standard method of measuring the IOP more than 60 years after its invention [16].

The concept of measuring the IOP intraocularly and continuously holds promise [24] and will certainly lead to further developments and new technologies, some of which appear to be on the horizon. For instance, a wireless implantable intraocular pressure monitor microsystem (IMM) that comprises a powering coil, an antenna, and a piezoresistive microelectromechanical system pressure sensor was recently described by Bhamra et al. That sensor, integrated on a 5 - $\mu \mathrm{m}$-thick biocompatible Parylene $C$ substrate, was reported by the authors to have been implanted into laboratory rodents [3]. Also, an optomechanical implant using an artificial neural network (ANN) has been implanted into rabbits, as reported by Kim et al. [15].

\section{MEDICAL THERAPY INITIATED BY SURGICAL PROCEDURE: INTRAOCULAR DRUG DELIVERY}

Visual field loss in glaucoma represents irreversible damage to visual function. It is crucial to diagnose and treat glaucoma in order to stop or at least slow the progression of the disease and thus delay additional optic nerve damage. The Achilles heel of conservative glaucoma therapy is the often less-than-stellar adherence. Many patients do not apply their antiglaucomatous eye drops correctly, as frequently as ordered, or at all. In a survey of 190 glaucoma patients in the United States who were taking one or more medications, $27 \%$ reported poor compliance. The most important risk factors for nonadherence in that study were decreased selfefficacy (odds ratio, OR: 4.7) and problems with administering the IOP-lowering eyedrops (OR 2.3). Other causes of insufficient adherence were forgetfulness (OR 5.6) and difficulties with the medication schedule (OR 2.9) [29]. There is a social imbalance in adherence: glaucoma patients who are more affluent, older, and white (compared to minority groups in the US population) tend to show better adherence [28]. In South Korea, one-third of the patients suffering from glaucoma were described as nonadherent, and the risk of failure to comply with the ophthalmologist's recommendations was 1.466 times greater for males than for females, and 1.328 -fold greater when the daily number of administrations was increased [14]. In Ethiopia, less than half of the glaucoma patients were considered nonadherent [22]. Nonadherence to pharmacological glaucoma therapy is a global issue.

The famous quote by the legendary US Surgeon General C. Everett Koop that drugs don't work in patients who do not take them fittingly describes a situation that is frustrating to many ophthalmologists. A way out of this dilemma is offered by sustained-release drug-delivery systems that are injected every few months by an ophthalmologist and which reduce the need for patient cooperation practically to zero. A number of such systems have been in clinical practice for some time; they contain steroids to treat different inflammatory processes. In addition to these previously established depots, several sustained-release systems for glaucoma therapy are currently investigational; at the time of writing none of these systems are FDA approved (a pilocarpine-releasing system named Ocusert is just a historic footnote, and was not injected into the eye but rather inserted under the lid, with a considerable degree of irritation in some patients). A sustained-release system that seems promising even though it does not meet the definition of miniaturization is the bimatoprost ocular ring; its long-term safety and efficacy have been reported by Brandt et al. [4].

Bimatoprost SR is a sustained-release system developed by Allergan that consists of a biodegradable implant and is injected into the anterior chamber with a 28-gauge applicator. In a 6-month phase I/II clinical trial, 75 glaucoma patients were administered Bimatoprost SR 
$(6 \mu \mathrm{g}, 10 \mu \mathrm{g}, 15 \mu \mathrm{g}$, or $20 \mu \mathrm{g})$ intracamerally in the study eye, with the fellow eye receiving topical bimatoprost $0.03 \%$ once daily. The IOP reduction from baseline to week 16 in the study eyes was $7.2,7.4,8.1$, and $9.5 \mathrm{mmHg}$ with the $6-\mu \mathrm{g}, 10-\mu \mathrm{g}, 15-\mu \mathrm{g}$, and $20-\mu \mathrm{g}$ dose strengths in the implant, respectively. The fellow eyes treated with topical bimatoprost showed an average IOP reduction of $8.4 \mathrm{mmHg}$. When adverse events occured, they tended to appear in study eyes within 2 days after the injection; in general they were transient. Immediately after the injection, the prevalence of conjunctival hyperemia was $24 \%$ in the study group, followed in prevalence by foreign body sensation (16\%) and eye pain (13.3\%). Given that local complications can be a factor in reduced adherence, it seems remarkable that later-onset conjunctival hyperemia was more frequent in eyes treated topically than in those that had received Bimatoprost SR $(17.3 \%$ vs $6.7 \%$ of eyes). Asked how likely they would be to recommend the procedure of receiving the bimatoprost implant by intraocular injection, $83 \%$ answered likely or very likely [19].

Another prostaglandin, travoprost, is injected intracamerally as a depot with the Envisia ENV515. This is a biodegradable polymer based on a new technology for nano- and microparticle formation. In a phase $2 \mathrm{a}$ open-label study over 28 days, an IOP reduction comparable to once-daily topical travoprost was achieved (relevant data were presented at AAO 2017; see the Acknowledgements section).

Slightly more invasive than just an injection is the procedure to place a depot drug-delivery system by the name of iDose in the appropriate position. iDose is a $1.8 \times 0.5 \mathrm{~mm}$ titanium implant containing a reservoir of travoprost capped by a membrane to allow continuous but gradual release. Just like the same manufacturer's iStent and iStent inject trabecular microbypass stent, iDose is surgically inserted into the trabecular meshwork and is supposed to be kept in place by a rather sharp scleral anchor at the peak of the small device. According to a press release by Glaukos, iDose was tested in a 154-patient, multicenter, randomized, double-blind phase II trial that was designed to evaluate two different versions of the iDose delivery system with two different elution rates of the IOP-lowering drug travoprost. Eyes in the control group received topical $0.5 \%$ timolol ophthalmic solution. The primary efficacy endpoint of the study was noninferiority to topical timolol. The phase II results were from an available interim cohort of 74 patients. An iDose Travoprost was implanted into 49 of them, while 25 patients were assigned to the timolol comparator group. Average IOP reductions observed in this cohort of implant patients during the first 12 months showed that iDose Travoprost achieved an approximate 30\% reduction in mean IOP vs. baseline IOP. After 12 months, the number of glaucoma medications ranged on average from 0.54 to 0.56 in the fast and slow iDose Travoprost elution implant groups, respectively. In the control group treated with topical timolol, the mean number of medications was 0.72 . There were reportedly no adverse events of hyperemia to date in either elution group (Glaukos press release, January 10, 2018).

While intraocular drug delivery systems hold promise for overcoming the adherence problem, it remains to be seen if patients are willing to undergo repeated injections. Besides concern about the repeated interventions, economic factors will probably figure prominently in the acceptance or rejection of this new form of treatment. In a recent survey in the US, $24 \%$ of participating glaucoma patients were ready to use such a new delivery system when the annual cost was $\$ 500$. Paying $\$ 1000$ per year reduced the proportion of patients willing to give intraocular drug depots a try to $18 \%$. Recommendation by an ophthalmologist increased the willingness to use such a system by $6-12 \%$; patients of an advanced age (65 years and older) and individuals with a low income were more likely to remain on eye drops [32].

\section{MINIMALLY INVASIVE GLAUCOMA SURGERY}

Over the last several years, glaucoma surgery has seen the addition of a number of procedures with a minimally invasive approach. The term MIGS (minimally invasive glaucoma surgery, 
sometimes termed micro-incisional glaucoma surgery) refers to a group of surgical procedures that have five distinct characteristics in common: a surgical approach that in most cases is ab interno (a new device, InnFocus, is inserted by an external approach) through a clear corneal incision which spares the conjunctiva from incision; does minimal damage to target tissue such as the trabecular meshwork; presents an IOP reduction efficacy that makes the technique a viable alternative to other more established procedures; has a good safety record that reduces the risk of serious complications; and facilitates a rapid recovery with minimal impact on the patient's quality of life [40].

Since MIGS was the subject of a recent major review in this journal [2], we will only outline a few general principles and share some significant data here. Basically, there are three groups of minimally incisional interventions: procedures that target the trabecular outflow; those that direct outflow into the suprachoroidal space; and interventions that create an opening under the conjunctiva, a filtering operation $a b$ interno.

Since the trabecular meshwork is considered the main point of resistance to aqueous humor outflow, bypassing this structure and directing the flow from the anterior chamber into Schlemm's canal seems to be a reasonable approach. It should be clear from planning such an intervention that the postoperative IOP cannot be lower than the episcleral venous pressure (EVP). The latter is not easy to evaluate but is reported in different studies to be in the range 7.6-9.1 $\mathrm{mmHg}$ [36]. The literature gives the impression that this approach probably has the greatest amount of published clinical experience. At the moment (and to the best of our current knowledge, given that newcomers appear quite rapidly in the field of MIGS), three stents for trabecular bypassing are available: iStent, iStent inject, and Hydrus. All three of them come with a specific injector system and have been implanted in a relatively large number of standalone procedures and as part of an extended cataract operation. iStent inject holds a special place among MIGSs, as it is the smallest of all the available microstents. It nevertheless seems to be able to lower IOP considerably.
In a recent study, two iStent injects were implanted in each of 99 eyes. Preoperatively, the mean IOP was $22.1 \pm 1.3 \mathrm{mmHg}$ (under topical antiglaucomatous medication) and $26.3 \pm 3.5 \mathrm{mmHg}$ (after a washout phase). Twelve months after the implantation of the iStent inject, the IOP had dropped by $40.2 \%$ compared to the baseline pressure and was reduced to a mean $15.7 \pm 3.7 \mathrm{mmHg}$. About a quarter of the study eyes required additional IOP reduction by topical medications. An IOP of $18 \mathrm{~mm} \mathrm{Hg}$ or lower with and without medication was achieved by $81 \%$ and $66 \%$ of the patients, respectively [44].

The Hydrus microstent is inserted through a clear corneal incision into Schlemm's canal. After the implantation, it dilates Schlemm's canal in the complete nasal quadrant. That mechanism causes the aqueous humor to bypass the trabecular meshwork. Pfeiffer et al. published a prospective, randomized, singlemasked 2-year clinical study comparing the efficacy and safety of a combined procedure with the Hydrus microstent implanted during cataract surgery and cataract surgery alone. Mean baseline IOPs were $26.3 \pm 4.4 \mathrm{mmHg}$ in the Hydrus plus cataract surgery study arm and $26.6 \pm 4.2 \mathrm{mmHg}$ in the phacoemulsification (PE/IOL) study arm. At the 24-month follow-up visit, mean IOPs were $16.9 \pm 3.3 \mathrm{mmHg}$ in the Hydrus/PE/IOL study arm and $19.2 \pm 4.7 \mathrm{mmHg}$ in the PE/IOL study arm. Twenty-four months after implantation, four out of five patients with Hydrus/PE/IOL experienced a decrease in IOP of $20 \%$ or more. This was achieved in a mere $46 \%$ of patients treated with PE/IOL only [35].

A device that creates outflow via the suprachoroidal space is the iStent Supra. It is CE approved and currently in the process of undergoing FDA approval. A prospective randomized trial involving more than 500 patients is currently under way across 36 sites. A recent publication reported on the effects of having one iStent Supra implanted in 80 patients together with two iStents. After 48 months, $97 \%$ and $98 \%$ of the eyes achieved IOP $\leq 15$ and $\leq 18 \mathrm{mmHg}$, respectively, on one medication [27]. 
Another option for MIGS is the subconjunctival approach. Not unlike in trabeculectomy, the aqueous humor is provided with a new, nonphysiological outflow. The XEN gel stent is inserted through a small, self-sealing, clear corneal incision. The device is brought into place in the subconjunctival space opposite the incision. Unlike the classical filtering operation, trabeculectomy, the procedure with the XEN does not disrupt the conjunctival and subconjunctival tissue. Just like in trabeculectomy, a bleb is created as a result of that new outflow. Pillunat et al. [36] have recommended the application of an antimetabolite such as mitomycin C (MMC) to prevent scar formation. Only one clinical study with the $45-\mu \mathrm{m}$ XEN gel stent has been published recently. The effectiveness and safety of phacoemulsification combined with the XEN implantation surgery were evaluated in patients with cataract and OAG in a prospective, 12-month follow-up study involving 30 eyes with at least two medications to control IOP. The mean preoperative IOP was $21.2 \pm 3.4 \mathrm{mmHg}$ with an average of 3.1 drugs. The study group reported an IOP reduction of $29.3 \%$ to an average IOP of $15.0 \mathrm{mmHg}$ after 12 months. This represents an average IOP reduction of $6.2 \mathrm{mmHg}$ [34]. This approach seemed to be particularly effective according to De Gregorio et al. [5] for the management of refractory glaucoma.

\section{DISCUSSION}

Glaucoma will remain a challenge for ophthalmologists and for health care systems everywhere. The number of glaucoma patients globally is expected to rise by 2020 to 65.5 million and by 2025 to 74.6 million, with men appearing to have a 33\% higher risk of developing POAG than women [13]. Wide segments of the population suffering from glaucoma are at risk of nonadherence when pharmacologically treated. Those considered most likely to exhibit nonadherence are patients in the age group 50-59 years, the oldest segment of the patient population $(80+$ years $)$, individuals who have suffered from glaucoma for a long period of time, and patients with considerable comorbidities, i.e., those suffering from three or more severe diseases in addition to glaucoma [9].

There have been many attempts to improve patient adherence, some of them with electronical monitoring, which in one observational study induced patients who used to take $75 \%$ or fewer of the prescribed doses to become more adherent (somewhat surprisingly, IOP did not correlate with adherence, and was not much different in the still poorly adherent control group and the successfully "treated" study group) [30]. Just recently, in a population with rather good therapy adherence in a society widely known for its high regard for discipline (Japan), a number of factors that influence this overall positive compliance were identified in a nationwide survey. These factors were the size of the clinic, the age and gender of the patients, the amounts and the types of IOP-lowering medications administered topically, the ease of instillation, the preferred number of eyedrops, the preferred frequency of eyedrop instillation, and the patient's awareness of and level of education about glaucoma [42]. As can easily be seen, none of these factors will come into play once an intraocular drug-delivery system is inserted, which is also the case for recently described factors such as forgetfulness, problems with handling the little bottles, etc.

Intraocular sustained-release drug-delivery systems seem to be an expanding market, and a number of companies are active in this field. While some data have been made public at meetings, there are only a limited number of publications on this promising technology. However, this approach demands repeated injections not unlike (though definitely less frequent than) those of current intravitreously injected VEGF inhibitors used in the treatment of a number of retinal diseases. Though such an injection seems to be the smallest invasive intervention possible in ophthalmology, the risk of an infection or other complications, though seemingly remote, cannot be completely ruled out. Another aspect is the patient's attitude (and possibly feelings such as fear, resentment, etc.) towards receiving injections every couple of months. 
There can be no doubt that MIGS has not only increased the number of available procedures that can lower IOP considerably, but that it has also opened up a pathway to surgical intervention for a particular and probably large segment of the glaucoma patient population. Less-invasive procedures have made surgical IOP lowering a realistic choice for patients (and their ophthalmologists) who shy away from a more complex procedure such as trabeculectomy [11]. MIGS has the potential to be an attractive alternative for patients who suffer from complications and discomfort after long periods of topical glaucoma therapy [8]. A profound pressure reduction which guarantees that the patient will no longer require antiglaucomatous eyedrops is generally not possible with MIGS. It is carried out to achieve a "safer" IOP level and to significantly reduce the number of medications required by the patient; in fact, a large percentage of patients require no medication following MIGS. The kind of success achievable with MIGS is illustrated by a study in which the percentage of patients with a Hydrus microstent plus cataract surgery who do not need additional IOP-lowering medications at month 24 (72.9\%) was significantly higher than the corresponding percentage in patients who underwent cataract surgery alone (37.8\%) [35].

MIGS is an emerging field, and the fact that so far no MIGS has become anything close to a "gold standard" seems to indicate that none of the three different approaches is vastly superior to the other two [39]. As Anzari has rightfully pointed out, there is currently an unsatisfied demand for high-quality data and concern because there is no standardization in most of the studies published on MIGS. Furthermore, there is a lack of cost-effectiveness data and long-term data and incomplete knowledge of ideal patient selection. In a very recent systematic literature review of nine randomized controlled trials (RCTs: seven on iStent, one on Hydrus, and one on CyPass), seven non-RCTs (three on iStent, three on CyPass, and one on Hydrus), as well as 23 economic studies, Agarwal et al. came to a somewhat cautious conclusion. They found that these devices tend to yield higher postoperative IOP levels than other approaches [45], and that subconjunctivally placed devices were associated with a relatively high rate of postoperative hypotony. They also found limited evidence for the cost-effectiveness of MIGS [1]. Furthermore, many studies of these small implants have focused on cases receiving both an implant and cataract surgery, meaning that they lack robust evidence for the effect of MIGS alone [2].

Nevertheless, the options described in this review have the potential to considerably increase the quality and efficacy of modern-day glaucoma management. However, a deeply sobering fact about glaucoma is that despite all the recent advances made in understanding the disease, in diagnostics, and in therapy, a number of our patients still progress to blindness [21]. Much has been done, but the quest for a better approach, with the ultimate goal being to halt the disease's progression, continues.

\section{CONCLUSION}

Successful management of glaucoma is based on a number of pillars. Based on our current and, in some cases, still rudimentary knowledge and limited clinical experience, the miniaturized technologies described in this review have the potential to improve glaucoma care. However, one major influence on the success of glaucoma therapy is not a device, a drug, or an intervention-it is a patient who is highly motivated to cooperate with the treatment by being well informed about the disease, preferably by his or her ophthalmologist. Because, to quote C. Everett Koop again, knowledge is the best prescription.

\section{ACKNOWLEDGEMENTS}

Funding. No funding or sponsorship was received for this study or the publication of this article.

Authorship. All named authors meet the International Committee of Medical Journal Editors (ICMJE) criteria for authorship for this manuscript, take responsibility for the integrity 
of the work as a whole, and have given final approval for the version to be published.

Prior Presentation. We benefited from and would like to express our appreciation for the splendid presentation by Jonathan S. Myers and Scott J. Fudemberg at the Annual Meeting of the American Academy of Ophthalmology (AAO) 2017 on intraocular drug-delivery systems.

Disclosures. H. Burkhard Dick, Tim Schultz, and Ronald D. Gerste have nothing to disclose.

Compliance and Ethics Guidelines. This article is based on previously conducted studies and does not contain any studies with human participants or animals performed by any of the authors.

Data Availability. The datasets obtained and/or analyzed during the current study are available from the corresponding author on reasonable request.

Open Access. This article is distributed under the terms of the Creative Commons Attribution-NonCommercial 4.0 International License (http://creativecommons.org/licenses/ by-nc/4.0/), which permits any noncommercial use, distribution, and reproduction in any medium, provided you give appropriate credit to the original author(s) and the source, provide a link to the Creative Commons license, and indicate if changes were made.

\section{REFERENCES}

1. Agrawal P, Bradshaw SE. Systematic literature review of clinical and economic outcomes of microinvasive glaucoma surgery (MIGS) in primary openangle glaucoma. Ophthalmol Ther. 2018;7:4973. https://doi.org/10.1007/s40123-018-0131-0.

2. Anzari E. An update on implants for minimally invasive glaucoma surgery (MIGS). Ophthalmol Ther. 2017;6:233-41.

3. Bhamra H, Tsai JW, Huang YW, Yuan Q, Shah JV, Irazoqui P. A subcubic millimeter wireless implantable intraocular pressure monitor microsystem. IEEE Trans Biomed Circuits Syst. 2017;11:1204-15.

4. Brandt JD, DuBiner HB, Benza R, Sall KN, Walker GA, Semba CP. Long-term safety and efficacy of a sustained-release bimatoprost ocular ring. Ophthalmology. 2017;124:1565-6.

5. De Gregorio A, Pedrotti E, Stevan G, Bertoncello A, Morselli S. XEN glaucoma treatment system in the management of refractory glaucomas: a short review on trial data and potential role in clinical practice. Clin Ophthalmol. 2018;12:773-82.

6. Dick HB, Schultz T. New developments in cataract surgery. Klin Monbl Augenheilkd. 2017;234:979-85 (article in German).

7. Flammer J, Mozaffarieh M. What is the present pathogenetic concept of glaucomatous optic neuropathy? Surv Ophthalmol. 2007;52(Suppl 2):S162-73.

8. Fingeret M, Dickerson JE Jr. The role of minimally invasive glaucoma surgery devices in the management of glaucoma. Optom Vis Sci. 2018;95:155-62.

9. Frech S, Kreft D, Guthoff RF, Doblhammer G. Pharmacoepidemiological assessment of adherence and influencing co-factors among primary openangle glaucoma patients-an observational cohort study. PLoS ONE. 2018;13(1):e0191185. https://doi. org/10.1371/journal.pone.0191185.

10. Gupta P, Zhao D, Guallar E, Ko F, Boland MV, Friedman DS. Prevalence of glaucoma in the United States: the 2005-2008 National Health and Nutrition Examination Survey. Investig Ophthalmol Vis Sci. 2016;57:2905-13.

11. Higginbotham EJ, Alexis D. Is newer necessarily better? The evolution of incisional glaucoma surgery over the last 100 years. Am J Ophthalmol. 2018;191:xxv-xxix. https://doi.org/10.1016/j.ajo. 2018.04.009.

12. Hoban K, Peden R, Megaw R, Halpin P, Tatham AJ. 24-Hour contact lens sensor monitoring of intraocular pressure-related profiles in normal-tension glaucoma and rates of disease progression. Ophthalmic Res. 2017;57:208-15.

13. Kapetanakis VV, Chan MP, Foster PJ, Cook DG, Owen CG, Rudnicka AR. Global variations and time trends in the prevalence of primary open angle glaucoma (POAG): a systematic review and metaanalysis. Br J Ophthalmol. 2016;100:86-93.

14. Kim CY, Park KH, Ahn J, et al. Treatment patterns and medication adherence of patients with glaucoma in South Korea. Br J Ophthalmol. 2017;101:801-7. 
15. Kim KH, Lee JO, Du J, Sretavan D, Choo H. Realtime in vivo intraocular pressure monitoring using an optomechanical implant and an artificial neural network. IEEE Sens J. 2017;17:7394-404.

16. Koutsonas A, Walter P, Plange N. Self-tonometry with a telemetric intraocular pressure sensor in patients with glaucoma. Klin Monbl Augenheilkd. 2016;233:743-8 (article in German).

17. Koutsonas A, Walter P, Roessler G, Plange N. Longterm follow-up after implantation of a telemetric intraocular pressure sensor in patients with glaucoma: a safety report. Clin Exp Ophthalmol. 2018:46(5):473-9. https://doi.org/10.1111/ceo. 13100.

18. Leidl MC, Choi CJ, Syed ZA, Melki SA. Intraocular pressure fluctuation and glaucoma progression: what do we know? $\mathrm{Br} \mathrm{J}$ Ophthalmol. 2014;98:1315-9.

19. Lewis RA, Christie WC, Day DG, Craven ER, Walters $\mathrm{T}$, Bejanian M, Lee SS, Goodkin ML, Zhang J, Whitcup SM, Robinson, Bimatoprost SR Study Group. Bimatoprost sustained-release implants for glaucoma therapy: 6-month results from a phase I/II clinical trial. Am J Ophthalmol. 2017;175:137-47.

20. Long E, Wan P, Zhuo Y. Predicting the real-world future of glaucoma patients? Cautions are required for machine learning. Transl Vis Sci Technol. 2017;6(6):3. https://doi.org/10.1167/tvst.6.6.3.

21. Malihi M, Moura Filho ER, Hodge DO, Sit AJ. Longterm trends in glaucoma-related blindness in Olmsted County, Minnesota. Ophthalmology. 2014;121:134-41.

22. Mehari T, Giorgis AT, Shibeshi W. Level of adherence to ocular hypotensive agents and its determinant factors among glaucoma patients in Menelik II Referral Hospital, Ethiopia. BMC Ophthalmol. 2016;2(16):131. https://doi.org/10.1186/s12886016-0316-z.

23. Melki S, Todani A, Cherfan G. An implantable intraocular pressure transducer: initial safety outcomes. JAMA Ophthalmol. 2014;132:1221-5.

24. Molaei A, Karamzadeh V, Safi S, Esfandiari H, Dargahi J, Khosravi MA. Upcoming methods and specifications of continuous intraocular pressure monitoring systems for glaucoma. J Ophthalmic Vis Res. 2018;13:66-71.

25. Mozaffarieh M, Flammer J. New insights in the pathogenesis and treatment of normal tension glaucoma. Curr Opin Pharmacol. 2013;13:43-9.
26. Mozaffarieh M, Grieshaber MC, Flammer J. Oxygen and blood flow: players in the pathogenesis of glaucoma. Mol Vis. 2008;14:224-33.

27. Myers JS, Masood I, Hornbeak DM, Belda JI, Auffarth G, Jünemann A, Giamporcaro JE, Martinez-dela-Casa JM, Ahmed IIK, Voskanyan L, Katz LJ. Prospective evaluation of two iStent ${ }^{\circledR}$ trabecular stents, one iStent Supra ${ }^{\circledR}$ suprachoroidal stent, and postoperative prostaglandin in refractory glaucoma: 4-year outcomes. Adv Ther. 2018;35:395-407.

28. Newman-Casey PA, Blachley T, Lee PP, Heisler M, Farris KB, Stein JD. Patterns of glaucoma medication adherence over four years of follow-up. Ophthalmology. 2015;122:2010-21.

29. Newman-Casey PA, Robin AL, Blachley T, Farris K, Heisler M, Resnicow K, Lee PP. The most common barriers to glaucoma medication adherence: a crosssectional survey. Ophthalmology. 2015;122:1308-16.

30. Okeke CO, Quigley HA, Jampel HD, Ying GS, Plyler RJ, Jiang Y, Friedman DS. Interventions improve poor adherence with once daily glaucoma medications in electronically monitored patients. Ophthalmology. 2009;116:2286-93.

31. Osorio-Alayo V, Pérez-Torregrosa VT, ClementeTomás R, Olate-Pérez Á, Cerdà-Ibáñez M, GargalloBenedicto A, Barreiro-Rego A, Duch-Samper A. Efficacy of the SENSIMED Triggerfish ${ }^{\circledR}$ in the postoperative follow-up of PHACO-ExPRESS combined surgery. Arch Soc Esp Oftalmol. 2017;92:372-8.

32. Ozdemir S, Wong TT, Allingham RR, Finkelstein EA. Predicted patient demand for a new delivery system for glaucoma medicine. Medicine (Baltim). 2017;96(15):e6626. https://doi.org/10.1097/md. 0000000000006626.

33. Paschalis EI, Cade F, Melki S, Pasquale LR, Dohlman $\mathrm{CH}$, Ciolino JB. Reliable intraocular pressure measurement using automated radio-wave telemetry. Clin Ophthalmol. 2014;8:177-85.

34. Pérez-Torregrosa VT, Olate-Pérez Á, Cerdà-Ibáñez $M$, et al. Combined phacoemulsification and XEN45 surgery from a temporal approach and 2 incisions. Arch Soc Esp Oftalmol. 2016;91:415-21.

35. Pfeiffer N, Garcia-Feijoo J, Martinez-de-la-Casa JM, et al. A randomized trial of a Schlemm's canal microstent with phacoemulsification for reducing intraocular pressure in open-angle glaucoma. Ophthalmology. 2015;122:1283-93.

36. Pillunat LE, Erb C, Jünemann AG, Kimmich F. Micro-invasive glaucoma surgery (MIGS): a review 
of surgical procedures using stents. Clin Ophthalmol. 2017;11:1583-600.

37. Quigley HA, Broman AT. The number of people with glaucoma worldwide in 2010 and 2020. Br J Ophthalmol. 2006;90:262-7.

38. Reitmeir P, Linkohr B, Heier M, et al. Common eye diseases in older adults of southern Germany: results from the KORA-Age study. Age Ageing. 2017;46:481-6.

39. Richter GM, Coleman AL. Minimally invasive glaucoma surgery: current status and future prospects. Clin Ophthalmol. 2016;10:189-206.

40. Saheb H, Ahmed II. Micro-invasive glaucoma surgery: current perspectives and future directions. Curr Opin Ophthalmol. 2012;23:96-104.

41. Sunaric-Megevand G, Leuenberger P, Preußner PR. Assessment of the Triggerfish contact lens sensor for measurement of intraocular pressure variations. Acta Ophthalmol. 2014;92:e414-5.
42. Tsumura T, Kashiwagi K, Suzuki Y, Yoshikawa K, Suzumura H, Maeda T, Takeda R, Saito H, Araie M. A nationwide survey of factors influencing adherence to ocular hypotensive eyedrops in Japan. Int Ophthalmol. 2018. https://doi.org/10.1007/ s10792-018-0820-7.

43. Vajaranant TS, Wu S, Torres M, Varma R. The changing face of primary open-angle glaucoma in the United States: demographic and geographic changes from 2011 to 2050. Am J Ophthalmol. 2012;154(303-314):e303.

44. Voskanyan L, García-Feijoó J, Belda JI, Fea A, Jünemann A, Baudouin C, Synergy Study Group. Prospective, unmasked evaluation of the iStent ${ }^{\circledR}$ inject system for open-angle glaucoma: synergy trial. Adv Ther. 2014;31:189-201.

45. Zetterström C, Behndig A, Kugelberg M, Montan P, Lundström M. Changes in intraocular pressure after cataract surgery: analysis of the Swedish National Cataract Register Data. J Cataract Refract Surg. 2015;41:1725-9. 